\title{
A Simpler Proof of the Negative Association Property for Absolute Values of Measures Tied to Generalized Orlicz Balls
}

by

\author{
Jakub Onufry WOJTASZCZYK
}

Presented by Stanistaw KWAPIEN

Summary. Negative association for a family of random variables $\left(X_{i}\right)$ means that for any coordinatewise increasing functions $f, g$ we have

$$
\mathbb{E} f\left(X_{i_{1}}, \ldots, X_{i_{k}}\right) g\left(X_{j_{1}}, \ldots, X_{j_{l}}\right) \leq \mathbb{E} f\left(X_{i_{1}}, \ldots, X_{i_{k}}\right) \mathbb{E} g\left(X_{j_{1}}, \ldots, X_{j_{l}}\right)
$$

for any disjoint sets of indices $\left(i_{m}\right),\left(j_{n}\right)$. It is a way to indicate the negative correlation in a family of random variables. It was first introduced in 1980s in statistics by Alem \& Saxena and Joag-Dev \& Proschan, and brought to convex geometry in 2005 by Wojtaszczyk \& Pilipczuk to prove the Central Limit Theorem for Orlicz balls.

The paper gives a relatively simple proof of negative association of absolute values for a wide class of measures tied to generalized Orlicz balls, including the uniform measures on such balls.

1. Introduction. We shall prove a property called the negative assoctiation of absolute values for a class of measures stemming from generalized Orlicz balls. The most important case, of uniform measures on generalized Orlicz balls, was considered in [6]. The proof given there, however, was complex and difficult to follow. The more general case, proved here, could probably also be tackled using the techniques from [6], but the paper would likely be even harder to read. The argument in this paper, using a technique similar to the Kannan-Lovász-Simonovits localization lemma, is much simpler. The result itself has quite a few consequences (see e.g. [6] or [2]); we will not explore them in this note. Negative association is defined as follows:

2000 Mathematics Subject Classification: Primary 52A20; Secondary 60D05.

Key words and phrases: negative association, Orlicz balls, log-concave measure. 
Definition 1.1. We say that a sequence $X_{1}, \ldots, X_{n}$ of random variables is negatively associated if for any bounded coordinatewise increasing functions $f: \mathbb{R}^{k} \rightarrow \mathbb{R}$ and $g: \mathbb{R}^{l} \rightarrow \mathbb{R}$ and any disjoint subsets $\left\{i_{1}, \ldots, i_{k}\right\}$ and $\left\{j_{1}, \ldots, j_{l}\right\}$ of $\{1, \ldots, n\}$ we have

$$
\operatorname{Cov}\left(f\left(X_{i_{1}}, \ldots, X_{i_{k}}\right), g\left(X_{j_{1}}, \ldots, X_{j_{l}}\right)\right) \leq 0 .
$$

This definition was introduced in the 1980s by Alam, Joag-Dev, Proschan and Saxena $([1],[4])$ for applications in statistics. It was applied in the context of convex geometry in [6] to prove the Central Limit Theorem for generalized Orlicz balls and in [2] to prove a reverse Hölder inequality for such balls.

In a linear space $V$ with a fixed basis $\left(e_{1}, \ldots, e_{n}\right)$, we denote by $x_{i}$ the coefficients of the basis expansion of a given vector $x$. We write $x \leq y$ for $x, y \in V$ if $x_{i} \leq y_{i}$ for all $i \in\{1, \ldots, \operatorname{dim} V\}$. Let $V_{+}$denote the set $\{x \in V: x \geq 0\}$, and $\operatorname{Int}_{l} K$ the relative interior of $K$ in $l$.

Recall the following definitions:

Definition 1.2. A Young function is an increasing, convex and lower semicontinuous function $f: \mathbb{R}_{+} \rightarrow \mathbb{R}_{+} \cup \infty$ with $f(0)=0$ and satisfying $f(x) \neq 0, f(y) \neq \infty$ for some $x, y>0$. A generalized Orlicz ball is a set in $\mathbb{R}^{n}$ given by the inequality $\sum_{i=1}^{n} f_{i}\left(\left|x_{i}\right|\right) \leq n$ for some Young functions $f_{1}, \ldots, f_{n}$.

As noted in [6], if $X$ is a random vector equidistributed on a 1 -symmetric convex body, one should consider the negative association property not for the sequence $\left(X_{i}\right)$, but rather for the absolute values $\left(\left|X_{i}\right|\right)$. For 1-symmetric bodies this is equivalent to considering random vectors equidistributed on the positive generalized quadrant of the body (that is, vectors conditioned by $X_{i} \geq 0$ for all $i$ ). Thus we shall work on $\mathbb{R}_{+}^{n}$ instead of $\mathbb{R}^{n}$.

The main result of this paper is the following

TheOREM 1.3. Let $f_{i}$ be Young functions and let $m: \mathbb{R}_{+} \rightarrow \mathbb{R}_{+}$be any log-concave nonincreasing function. Assume that the measure on $\mathbb{R}^{n}$ with density $m\left(\sum f_{i}\left(\left|x_{i}\right|\right)\right)$ is a probability measure and let $X$ be a random vector distributed according to this measure. Then the sequence $\left|X_{1}\right|, \ldots,\left|X_{n}\right|$ is negatively associated.

In particular, we recover the negative association of absolute values for random vectors uniformly distributed on generalized Orlicz balls by taking $m=\mathbf{1}_{[0, n]}$.

1.1. Notation. Note that the property of being an Orlicz ball depends upon the choice of the coordinate system (or basis) in the space, and thus one should rather say that a set is an Orlicz ball in a given coordinate system. We shall mostly use the language of functions (that is, instead of 
the Orlicz ball, we consider its characteristic function), which motivates the following definitions:

DeFinition 1.4. By an oriented function we shall mean a triple $\mathcal{F}=$ $(s, V, \mathcal{E})$, where $V$ is a linear space of finite dimension over $\mathbb{R}, \mathcal{E}$ is a basis of $V$ and $s: V_{+} \rightarrow \mathbb{R}$.

Definition 1.5. An oriented function $\mathcal{F}=(s, V, \mathcal{E})$ is Orlicz-based if

$$
s\left(x_{1}, \ldots, x_{n}\right)=m\left(\sum_{i=1}^{n} f_{i}\left(x_{i}\right)\right) \prod_{i=1}^{n} w_{i}\left(x_{i}\right),
$$

where $\left(f_{i}\right)_{i=1}^{n}$ are Young functions or $f_{i} \equiv \infty,\left(w_{i}\right)_{i=1}^{n}$ are log-concave, upper semicontinuous functions supported on $\mathbb{R}_{+}$, and $m: \mathbb{R}_{+} \cup\{\infty\} \rightarrow \mathbb{R}_{+}$is a log-concave upper semicontinuous function with compact support, attaining its maximum at 0 , with $m(\infty)=0$.

Note that an Orlicz-based function is continuous on its support, although it may have a discontinuity on the boundary. We shall sometimes speak of $s$ as being a function on the whole of $V$ by extending it by 0 outside $V_{+}$.

The characteristic function of the positive generalized quadrant of a generalized Orlicz ball gives the simplest example of an Orlicz-based function.

2. A localization type lemma. The idea given below is similar to the so-called localization lemma proven in [5]. It allows us to circumvent the transfinite induction used in the original proof in [6].

The crucial property of the class of Orlicz-based functions is that it is closed under the transformations defined below.

Definition 2.1. Let $\mathcal{F}=(s, V, \mathcal{E})$ be an oriented function. Then we define the sons of $\mathcal{F}$ as follows:

- For $i \in\{1, \ldots, \operatorname{dim} V\}$ and a log-concave, upper semicontinuous function $w: \mathbb{R}_{+} \rightarrow \mathbb{R}_{+}$the triple $(\widetilde{s}, V, \mathcal{E})$ is a son of $\mathcal{F}$, where $\widetilde{s}(x)=$ $s(x) \cdot w\left(x_{i}\right)$.

- If $H$ is an affine hyperplane in $V$ given by the equation $x_{i}=a x_{j}+b$ for some nonnegative $a, b$ and $i, j \in\{1, \ldots, \operatorname{dim} V\}$, then the triple $(\widetilde{s}, \widetilde{H}, \widetilde{\mathcal{E}})$ is a son of $\mathcal{F}$, where $\widetilde{H}$ is defined to be $H$ with the linear structure given by moving the origin to the point $x_{i}=b, x_{k}=0$ for $k \neq i ; \widetilde{\mathcal{E}}$ is obtained from $\mathcal{E}$ by substituting $a e_{i}+e_{j}$ for the pair $e_{i}, e_{j} ;$ and $\widetilde{s}$ is the restriction of $s$ to $\widetilde{H}$.

- If $x \in V_{+}$, then the triple $(\widetilde{s}, \widetilde{V}, \mathcal{E})$ is a son of $\mathcal{F}$, where $\widetilde{V}$ is $V$ with origin at $x$, and $\widetilde{s}$ is $s$ restricted to $\widetilde{V}$.

We define the relation of being a descendant of an oriented function $\mathcal{F}$ as the smallest transitive and reflexive extension of the relation of being a son. 
Lemma 2.2. Let $\mathcal{F}=(s, V, \mathcal{E})$ be an Orlicz-based function. Then any descendant of $\mathcal{F}$ is also an Orlicz-based function.

Proof. It suffices to consider any son $\mathcal{F}^{\prime}$ of $\mathcal{F}$. Let the functions $f_{i}, w_{i}$ and $m$ give a representation of $\mathcal{F}$ as an Orlicz-based function. We shall define functions $f_{i}^{\prime}, w_{i}^{\prime}$ and $m^{\prime}$ which represent $\mathcal{F}^{\prime}$ as an Orlicz-based function. In the first case of the definition (where $\mathcal{F}^{\prime}$ is formed by multiplying $w_{i}$ by $w)$ we take $f_{i}^{\prime}=f_{i}, m^{\prime}=m$ and $w_{j}^{\prime}=w_{j}$ for $j \neq i$, while $w_{i}^{\prime}=w \cdot w_{i}$. In the second case it suffices to replace the pair $f_{i}, f_{j}$ by a single function $f_{i}(a t+b)+f_{j}(t)-f_{i}(b)$ (we assume $\infty-\infty=\infty$ ), analogously replace $w_{i}$, $w_{j}$ by a single $w$ and put $m^{\prime}(t)=m\left(t+f_{i}(b)\right)$. In the third case, we take $f_{i}^{\prime}(t)=f_{i}\left(t+x_{i}\right)-f_{i}\left(x_{i}\right), w_{i}^{\prime}(t)=w_{i}\left(t+x_{i}\right)$ and $m^{\prime}(t)=m\left(t+\sum f_{i}\left(x_{i}\right)\right)$.

We begin by observing a simple geometric fact:

LEMma 2.3. Let $\left(K_{m}\right)$ be a descending sequence of compact, convex sets of dimension $k$ in $\mathbb{R}^{k}$, and let $K=\bigcap_{m=1}^{\infty} K_{m}$. Let $l$ be the affine subspace of dimension $p:=\operatorname{dim} K$ spanned by $K$. Let $g_{m}(x)=\lambda_{k-p}\left(K_{m} \cap P^{-1}(x)\right) /$ $\lambda_{k}\left(K_{m}\right)$ for $x \in l$, where $P$ is the orthogonal projection to $l$. Then there exists a subsequence $K_{m_{i}}$ of $K_{m}$ and a log-concave function $g$ on $l$ with support $K$ such that $g_{m_{i}}$ converges almost uniformly to $g$ on $\operatorname{Int}_{l} K$ and $\int_{K} g=1$. Moreover, the family $\left(g_{m}\right)$ is uniformly bounded on $l$.

Proof. If $p=k$, then $\left(g_{m}\right)$ converges uniformly on $K$ to $\mathbf{1}_{K} / \lambda_{k}(K)$. Further on we assume $p<k$.

Let $T_{m}$ denote the maximum of $g_{m}$ on $l$; suppose it is attained at the point $O$. Let $\phi_{m}$ be the Minkowski functional on $l$ given by supp $g_{m}$, where $O$ is taken to be the origin (that is, $\phi_{m}(x)=\inf \left\{\lambda: O+(x-O) / \lambda \in \operatorname{supp} g_{m}\right\}$ ). The function $g_{m}$ is the density of the projection of a uniform measure on a $k$-dimensional convex set to a $p$-dimensional subspace, thus by the BrunnMinkowski inequality (see e.g. [3]) $\sqrt[k-p]{g_{m}}$ is concave on its support, so if $\phi_{m}(x) \leq 1$, then $g_{m}(x) \geq T_{m}\left(1-\phi_{m}(x)\right)^{k-p}$. Further,

$$
\begin{aligned}
1 & =\int_{l} g_{m}(x) d \lambda_{p}(x)=\int_{0}^{T_{m}} \lambda_{p}\left\{x: g_{m}(x) \geq t\right\} d t \\
& \geq \int_{0}^{T_{m}} \lambda_{p}\left\{x: T_{m}\left(1-\phi_{m}(x)\right)^{k-p} \geq t\right\} d t \\
& =\int_{0}^{T_{m}} \lambda_{p}\left\{x: \phi_{m}(x) \leq 1-\left(\frac{t}{T_{m}}\right)^{1 /(k-p)}\right\} d t \\
& =\int_{0}^{T_{m}} \lambda_{p}\left(\left(1-\left(\frac{t}{T_{m}}\right)^{1 /(k-p)}\right) \operatorname{supp} g_{m}\right) d t
\end{aligned}
$$




$$
\begin{aligned}
& =\int_{0}^{T_{m}}\left(1-\left(\frac{t}{T_{m}}\right)^{1 /(k-p)}\right)^{p} \lambda_{p}\left(\operatorname{supp} g_{m}\right) d t \\
& =T_{m} \lambda_{p}\left(\operatorname{supp} g_{m}\right) \int_{0}^{1}\left(1-s^{1 /(k-p)}\right)^{p} d s \\
& =T_{m} \lambda_{p}\left(\operatorname{supp} g_{m}\right) c_{k, p} \geq T_{m} \lambda_{p}(K) c_{k, p} .
\end{aligned}
$$

Hence $T_{m} \leq 1 /\left(c_{k, p} \lambda_{p}(K)\right)$, and so the sequence $g_{m}$ is uniformly bounded.

Our aim is to apply the Arzelà-Ascoli theorem, so we need to prove almost uniform equicontinuity, that is, uniform equicontinuity on any compact subset $L$ of $\operatorname{Int}_{l} K$. By compactness we can choose a $\delta$ such that $|x-z|>\delta$ for any $x \in L$ and $z \notin K$.

Fix $\Gamma>1$. For any $x, y \in L$ with $|x-y|<\delta / \Gamma$ we can choose a $z \in K$ such that $\Gamma y=(\Gamma-1) x+z$. Then

$$
\begin{aligned}
g_{m}(x)^{1 /(k-p)}-\frac{T_{m}^{1 /(k-p)}}{\Gamma} & \leq \frac{\Gamma-1}{\Gamma} g_{m}(x)^{1 /(k-p)} \\
& \leq \frac{\Gamma-1}{\Gamma} g_{m}(x)^{1 /(k-p)}+\frac{1}{\Gamma} g_{m}(z)^{1 /(k-p)} \\
& \leq g_{m}(y)^{1 /(k-p)},
\end{aligned}
$$

and thus

$$
\begin{aligned}
g_{m}(y) & -g_{m}(x) \\
\leq & \frac{T_{m}^{1 /(k-p)}}{\Gamma}\left(g_{m}(y)^{\frac{k-p-1}{k-p}}+g_{m}(y)^{\frac{k-p-2}{k-p}} g_{m}(x)+\cdots+g_{m}(x)^{\frac{k-p-1}{k-p}}\right) \\
& \leq \frac{(k-p) T_{m}}{\Gamma} \leq \frac{k-p}{\Gamma c_{k, p} \lambda_{p}(K)} .
\end{aligned}
$$

This expression is independent of $m, x$ and $y$, and by choosing a sufficiently large $\Gamma$ we can make it arbitrarily small, thus indeed the sequence $\left(g_{m}\right)$ on $L$ is uniformly equicontinuous. Thus by the Arzelà-Ascoli theorem we can choose a subsequence of $g_{m}$ uniformly convergent on $L$. By choosing a sequence of $L$ 's increasing to $K$ we can diagonally construct a subsequence $\left(g_{m_{i}}\right)$ almost uniformly convergent on $K$. Let $g$ be the limit of $g_{m_{i}}$ on $\operatorname{Int}_{l} K$, extended outside by 0 . As the $g_{m}$ are uniformly bounded, by the Lebesgue dominated convergence theorem we have $\int_{K} g_{m} \rightarrow \int_{K} g$; on the other hand, as $\bigcap K_{m}=K$ and the $g_{m}$ are uniformly bounded, $\int_{K} g_{m} \rightarrow 1$, thus $\int_{K} g=1$. Sine all $g_{m}$ 's are log-concave, by a simple limit argument $g$ is also log-concave on $\operatorname{Int}_{l} K$, which ends the proof.

COROLlary 2.4. Let $K_{m}$ be a descending sequence of compact, convex sets of dimension $k$ in $\mathbb{R}^{k} \subset \mathbb{R}^{n}$, and let $K=\bigcap K_{m}$. Then there exists a log-concave function $g$ on $K$ and a subsequence $K_{m_{i}}$ of $K_{m}$ such that for 
any continuous, compactly supported $f$ on $\mathbb{R}^{n}$ we have

$$
\frac{\int_{K_{m_{i}} \times \mathbb{R}^{n-k}} f(v) d \lambda_{n}(v)}{\lambda_{k}\left(K_{m_{i}}\right)} \underset{m_{i} \rightarrow \infty}{\longrightarrow} \int_{K \mathbb{R}^{n-k}} \int_{1} f(x, y) g(x) d \lambda_{n-k}(y) d \lambda_{\operatorname{dim} K}(x) .
$$

The same holds if $f$ has a compact, convex support, and is continuous on its support, while $K \times \mathbb{R}^{n-2}$ contains a point from the interior of $\operatorname{supp} f$.

Proof. Let $p=\operatorname{dim} K$, and let $l$ be the affine subspace of dimension $p$ spanned by $K$. Let $P$ be the orthogonal projection from $\mathbb{R}^{n}$ onto $l \times \mathbb{R}^{n-k}$, and set $g_{m}(x)=\lambda_{k-p}\left(K_{m} \cap P^{-1}(x)\right) / \lambda_{k}\left(K_{m}\right)$ for $x \in l$. Take the sequence $m_{i}$ and the function $g$ on $K$ according to Lemma 2.3 so that $g_{m_{i}}$ converges to $g$ almost uniformly on $\operatorname{Int}_{l} K$. For simplicity we assume $g_{m}$ itself converges almost uniformly to $g$ on $\operatorname{Int}_{l} K$. Now

$$
\begin{aligned}
& \frac{\int_{K_{m} \times \mathbb{R}^{n-k}} f(v) d \lambda_{n}(v)}{\lambda_{k}\left(K_{m}\right)} \\
& =\frac{\int_{l} \int_{\mathbb{R}^{n-k}} \int_{K_{m} \cap P^{-1}(x)} f(x, y, z) d \lambda_{k-p}(z) d \lambda_{n-k}(y) d \lambda_{p}(x)}{\lambda_{k}\left(K_{m}\right)} \\
& =\int_{l \mathbb{R}^{n-k}} g_{m}(x) \frac{\int_{K_{m} \cap P^{-1}(x)} f(x, y, z) d \lambda_{k-p}(z)}{\lambda_{k-p}\left(K_{m} \cap P^{-1}(x)\right)} d \lambda_{n-k}(y) d \lambda_{p}(x) .
\end{aligned}
$$

Let

$$
f_{m}(x, y)= \begin{cases}\frac{\int_{K_{m} \cap P^{-1}(x)} f(x, y, z) d \lambda_{k-p}(z)}{\lambda_{k-p}\left(K_{m} \cap P^{-1}(x)\right)}, & \lambda_{k-p}\left(K_{m} \cap P^{-1}(x)\right)>0, \\ f(x, y, 0), & \lambda_{k-p}\left(K_{m} \cap P^{-1}(x)\right)=0 .\end{cases}
$$

Note that as the $K_{m}$ are compact, $\lim _{m \rightarrow \infty} \sup _{v \in K_{m}} \operatorname{dist}(v, K)=0$, thus in particular the diameter of $K_{m} \cap P^{-1}(x)$ converges to zero uniformly with respect to $x$. Meanwhile $f$ is continuous and compactly supported, so $f_{m}(x, y)$, being the average of a uniformly continuous function over a set with diameter uniformly converging to zero, converges uniformly to $f(x, y, 0)$. It is also uniformly bounded (as $f$ is bounded).

Since $g_{m}$ 's are uniformly bounded and almost uniformly convergent on $K$, the product $f_{m}(x, y) g_{m}(x)$ is almost uniformly convergent on $K \times \mathbb{R}^{n-k}$. As $\lambda_{p}\left(\operatorname{supp} g_{m} \backslash K\right) \rightarrow 0$, while $f_{m}$ is compactly supported and bounded, we have

$$
\begin{aligned}
\frac{\int_{K_{m} \times \mathbb{R}^{n-k}} f(v) d \lambda_{n}(v)}{\lambda_{k}\left(K_{m}\right)} & =\iint_{l \mathbb{R}^{n-k}} g_{m}(x) f_{m}(x, y) d \lambda_{n-k}(y) d \lambda_{p}(x) \\
& =\int_{l \backslash K \mathbb{R}^{n-k}} \int_{m} g_{m}(x) f_{m}(x, y) d \lambda_{n-k}(y) d \lambda_{p}(x)
\end{aligned}
$$




$$
\begin{array}{r}
+\int_{K} \int_{\mathbb{R}^{n-k}} g_{m}(x) f_{m}(x, y) d \lambda_{n-k}(y) d \lambda_{p}(x) \\
\underset{m \rightarrow \infty}{\longrightarrow} 0+\int_{K} \int_{\mathbb{R}^{n-k}} g(x) f(x, y, 0) d \lambda_{n-k}(y) d \lambda_{p}(x) .
\end{array}
$$

For the second statement, let $O \in\left(K \times \mathbb{R}^{n-2}\right) \cap \operatorname{Int} \operatorname{supp} f$, and assume $O$ is the origin for the simplicity of notation. Let $f_{\varepsilon}$ be a continuous function equal to $f$ on $(1-\varepsilon) \operatorname{supp} f, 0$ outside supp $f$ and between sup $|f|$ and $-\sup |f|$ in between. Now

$$
\begin{gathered}
\frac{\int_{K_{m} \times \mathbb{R}^{n-2}}\left|f-f_{\varepsilon}\right|}{\lambda_{k}\left(K_{m}\right)} \leq \frac{\int_{K_{m} \times \mathbb{R}^{n-2}}\left|f-f_{\varepsilon}\right| \lambda_{n-k}\left(P_{n-k}(\operatorname{supp} f)\right)}{\lambda_{n}\left(\left(K_{m} \times \mathbb{R}^{n-k}\right) \cap \operatorname{supp} f\right)} \\
\leq 2 \sup |f| \lambda_{n-k}\left(P_{n-k}(\operatorname{supp} f)\right) \frac{\lambda_{n}\left\{x \in K_{m} \times \mathbb{R}^{n-k}: f(x) \neq f_{\varepsilon}(x)\right\}}{\lambda_{n}\left(\left(K_{m} \times \mathbb{R}^{n-k}\right) \cap \operatorname{supp} f\right)},
\end{gathered}
$$

where $P_{n-k}$ denotes the orthogonal projection onto $\mathbb{R}^{n-k}$. Now

$$
\begin{aligned}
\left\{x \in K_{m}\right. & \left.\times \mathbb{R}^{n-k}: f(x) \neq f_{\varepsilon}(x)\right\} \\
& \subset\left(\left(K_{m} \times \mathbb{R}^{n-k}\right) \cap \operatorname{supp} f\right) \backslash\left(\left(K_{m} \times \mathbb{R}^{n-k}\right) \cap(1-\varepsilon) \operatorname{supp} f\right) \\
& \subset\left(\left(K_{m} \times \mathbb{R}^{n-k}\right) \cap \operatorname{supp} f\right) \backslash(1-\varepsilon)\left(\left(K_{m} \times \mathbb{R}^{n-k}\right) \cap \operatorname{supp} f\right) .
\end{aligned}
$$

The measure of the last set is $\left(1-(1-\varepsilon)^{n}\right) \lambda_{n}\left(\left(K_{m} \times \mathbb{R}^{n-k}\right) \cap\right.$ supp $\left.f\right)$, which gives us a uniform bound in (2) which tends to 0 as $\varepsilon \rightarrow 0$. By the first part of the corollary we have the statement for each $f_{\varepsilon}$, and the error term tends to 0 as $\varepsilon \rightarrow 0$ uniformly in $m$; thus we get the statement for $f$.

We now proceed to the localization lemma. The following definitions will be useful:

Definition 2.5. A set $K \subset \mathbb{R}^{2}$ is called spanned by the points $a, b$ if $K$ is convex, compact, $a, b \in K$, and for any $x \in K$ we have $a \leq x \leq b$. A set is called spanned if it is spanned by some two points $a, b$.

Geometrically this definition means that $K$ is convex, compact and if we inscribe $K$ in a rectangle with edges parallel to the coordinate axes, then the lower left corner and the upper right corner of the rectangle are contained in $K$.

Definition 2.6. For a linear space $V$ with a basis $\mathcal{E}$, by a splitting of $V$ with respect to $\mathcal{E}$ we mean a decomposition $V=V_{1} \oplus V_{2}$ and $\mathcal{E}=\mathcal{E}_{1} \cup \mathcal{E}_{2}$ such that $\mathcal{E}_{i}$ is a basis of $V_{i}$.

Definition 2.7. Consider an Orlicz-based function $\mathcal{F}=(s, V, \mathcal{E})$ and functions $f, g: V \rightarrow \mathbb{R}$. We shall say that $\mathcal{F}, f$ and $g$ satisfy the $\Theta$ condition if for any splitting $V=V_{1} \oplus V_{2}$ with respect to $\mathcal{E}$ and any $0 \leq x \leq y \in V_{1}$ 
we have

$$
\frac{\int_{V_{2}} f(x, z) s(x, z) d \lambda_{k}(z)}{\int_{V_{2}} g(x, z) s(x, z) d \lambda_{k}(z)} \geq \frac{\int_{V_{2}} f(y, z) s(y, z) d \lambda_{k}(z)}{\int_{V_{2}} g(y, z) s(y, z) d \lambda_{k}(z)},
$$

whenever both sides are well defined, where $k$ denotes $\operatorname{dim} V_{2}$.

We shall say that an Orlicz-based function $\mathcal{F}$ and functions $f, g$ satisfy the hereditary $\Theta$ condition if any descendant $\mathcal{F}^{\prime}=\left(s^{\prime}, V^{\prime}, \mathcal{E}^{\prime}\right)$ of $\mathcal{F}$ and the restrictions of $f$ and $g$ to $V^{\prime}$ satisfy the $\Theta$ condition.

LEMma 2.8. Consider an Orlicz-based function $\mathcal{F}=\left(s, \mathbb{R}^{n}, \mathcal{E}\right)$, where $\mathcal{E}$ is the standard basis in $\mathbb{R}^{n}$, and three continuous functions: $f, g: \mathbb{R}^{n} \rightarrow$ $[0, M]$ and $h: \mathbb{R}^{n} \rightarrow[\varepsilon, M]$ for some $M>\varepsilon>0$, where $\{f>0\} \cup\{s>0\} \subset$ $\{g>0\}$ and $\int_{\mathbb{R}^{n}} s(x) d x>0$. Assume that $\mathcal{F}, f$ and $g$ satisfy the hereditary $\Theta$ condition. Additionally assume that $f$ has a convex support and that

$$
\frac{\int_{\mathbb{R}_{+}^{n}} f(x) h(x) s(x) d z}{\int_{\mathbb{R}_{+}^{n}} g(x) h(x) s(x) d z}<\frac{\int_{\mathbb{R}_{+}^{n}} f(x) s(x) d z}{\int_{\mathbb{R}_{+}^{n}} g(x) s(x) d z} .
$$

Then there exist two different points $a \leq b \in \mathbb{R}_{+}^{n}$ and a log-concave measure $\nu$ on the interval $I=[a, b]$ such that

$$
\frac{\int_{\mathbb{R}_{+}^{n}} f(x) s(x) d x}{\int_{\mathbb{R}_{+}^{n}} g(x) s(x) d x}=\frac{\int_{I} f(x) s(x) d \nu(x)}{\int_{I} g(x) s(x) d \nu(x)}>\frac{\int_{I} f(x) h(x) s(x) d \nu(x)}{\int_{I} g(x) h(x) s(x) d \nu(x)} .
$$

In particular, $h$ cannot be coordinatewise nonincreasing.

Proof. We shall proceed by induction upon dimension. For $n=0$ the condition (4) cannot be satisfied. For $n=1$ no assumptions are needed, the interval supp $s$ with the Lebesgue measure satisfies the conclusion of the lemma. Let us consider higher $n$. We shall a construct a decreasing sequence of spanned sets $K_{0} \supset K_{1} \supset \cdots$ in $\operatorname{span}\left\{e_{1}, e_{2}\right\}$ satisfying the following four conditions:

$$
\begin{gathered}
\int_{K_{m} \times \mathbb{R}_{+}^{n-2}} s(x) d x>0 \\
\frac{\int_{K_{m} \times \mathbb{R}_{+}^{n-2} f(x) s(x) d x}}{\int_{K_{m} \times \mathbb{R}_{+}^{n-2}} g(x) s(x) d x}=\frac{\int_{\mathbb{R}_{+}^{n}} f(x) s(x) d x}{\int_{\mathbb{R}_{+}^{n}} g(x) s(x) d x} \\
\frac{\int_{K_{m} \times \mathbb{R}_{+}^{n-2}} f(x) h(x) s(x) d x}{\int_{K_{m} \times \mathbb{R}_{+}^{n-2}} g(x) h(x) s(x) d x} \leq \frac{\int_{K_{m} \times \mathbb{R}_{+}^{n-2}} f(x) h(x) s(x) d x}{\int_{K_{m} \times \mathbb{R}_{+}^{n-2}} g(x) h(x) s(x) d x} \\
\leq \frac{\int_{\mathbb{R}_{+}^{n}} \tilde{f}(x) h(x) s(x) d x}{\int_{\mathbb{R}_{+}^{n}} g(x) h(x) s(x) d x}<\frac{\int_{\mathbb{R}_{+}^{n}} f(x) s(x) d x}{\int_{\mathbb{R}_{+}^{n}} g(x) s(x) d x}, \\
\bigcap_{m=0}^{\infty} K_{m} \text { is an interval or a point. }
\end{gathered}
$$


The function $\tilde{f}$ is a slight modification of $f$, which ensures that our sequence does not approach the edge of supp $s$ too closely. Choose $M^{\prime}$ so that

$$
\frac{M^{\prime}}{M}>\frac{\int_{\mathbb{R}_{+}^{n}} f(x) s(x) d x}{\int_{\mathbb{R}_{+}^{n}} g(x) s(x) d x}
$$

and $\widetilde{c}>0$ so that

$$
\frac{\widetilde{c} M^{\prime} M+\int_{\mathbb{R}_{+}^{n}} f(x) h(x) s(x) d x}{\int_{\mathbb{R}_{+}^{n}} g(x) h(x) s(x) d x}<\frac{\int_{\mathbb{R}_{+}^{n}} f(x) s(x) d x}{\int_{\mathbb{R}_{+}^{n}} g(x) s(x) d x} .
$$

Let

$$
A_{\varepsilon}:=\left\{x: s(x) \leq \varepsilon \vee \operatorname{dist}\left(x, \mathbb{R}^{n} \backslash \operatorname{supp} s\right) \leq \varepsilon\right\} .
$$

Fix $t>0$ so that $\int_{A_{t}} s(x) d x<\widetilde{c}$. Let $\Delta f$ be a continuous function which is equal to $M^{\prime}$ on $A_{t / 2}$, equal to 0 on $\mathbb{R}_{+}^{n} \backslash A_{t}$ and is bounded from below by zero, and from above by $M^{\prime}$. Then $\widetilde{f}:=f+\Delta f$. Notice that the third inequality of condition (7) is satisfied by (10), the first inequality is satisfied as $\Delta f$ is nonnegative, and if $L$ is a set contained in $A_{t / 2}$ then by (9) and $(10)$,

$$
\frac{\int_{L} \tilde{f}(x) h(x) s(x) d x}{\int_{L} g(x) h(x) s(x) d x} \geq \frac{M^{\prime}}{M}>\frac{\int_{\mathbb{R}_{+}^{n}} f(x) s(x) d x}{\int_{\mathbb{R}_{+}^{n}} g(x) s(x) d x}>\frac{\int_{\mathbb{R}_{+}^{n}} \tilde{f}(x) h(x) s(x) d x}{\int_{\mathbb{R}_{+}^{n}} g(x) h(x) s(x) d x}
$$

For $K_{0}$ we can take any rectangle in $\operatorname{span}\left\{e_{1}, e_{2}\right\}$ with edges parallel to the coordinate axes and containing the projection of $\operatorname{supp} s$ onto $\operatorname{span}\left\{e_{1}, e_{2}\right\}$. We order all the points with both coordinates rational into a sequence $\left(q_{i}\right)_{i=1}^{\infty}$. Having $K_{m}$ we now construct $K_{m+1}$. Let $O_{m}$ be the first point from the sequence $\left(q_{i}\right)$ contained in the interior of $K_{m}$ (by (5), $K_{m}$ is a convex set of positive measure, and thus contains a point with both coordinates rational). Consider a vertical (i.e. parallel to $e_{2}$ ) line passing through $O_{m}$, and denote by $K_{E}$ the part of $K_{m}$ to the right of that line, and by $K_{W}$ the part to the left. Lemma 2.9 below shows that under the present assumptions we have

$$
\frac{\int_{K_{W} \times \mathbb{R}_{+}^{n-2}} f(x) s(x) d x}{\int_{K_{W} \times \mathbb{R}_{+}^{n-2}} g(x) s(x) d x} \geq \frac{\int_{K_{E} \times \mathbb{R}_{+}^{n-2}} f(x) s(x) d x}{\int_{K_{E} \times \mathbb{R}_{+}^{n-2}} g(x) s(x) d x}
$$

if both sides are well defined. If one of the sides is not well defined (say the one corresponding to $\left.K_{E}\right)$, then $\left(K_{E} \times \mathbb{R}_{+}^{n-2}\right) \cap \operatorname{supp} s$ has measure zero, so we can set $K_{m+1}=K_{W}$-all integrals on $K_{W} \times \mathbb{R}_{+}^{n-2}$ will be equal to the corresponding integrals on $K_{m} \times \mathbb{R}_{+}^{n-2}$, and as $K_{m}$ satisfies (5)-(7), so also does $K_{W}$. We shall check condition (8) later. Thus assume both sides are 
well defined. Then

$$
\frac{\int_{K_{E} \times \mathbb{R}_{+}^{n-2}} f(x) s(x) d x}{\int_{K_{E} \times \mathbb{R}_{+}^{n-2}} g(x) s(x) d x} \leq \frac{\int_{K_{m} \times \mathbb{R}_{+}^{n-2}} f(x) s(x) d x}{\int_{K_{m} \times \mathbb{R}_{+}^{n-2}} g(x) s(x) d x}=\frac{\int_{\mathbb{R}_{+}^{n}} f(x) s(x) d x}{\int_{\mathbb{R}_{+}^{n}} g(x) s(x) d x} .
$$

Similarly, when we consider a horizontal line through $O_{m}$, dividing $K_{m}$ into the upper part $K_{N}$ and lower part $K_{S}$, Lemma 2.9 gives

$$
\frac{\int_{K_{S} \times \mathbb{R}_{+}^{n-2}} f(x) s(x) d x}{\int_{K_{S} \times \mathbb{R}_{+}^{n-2}} g(x) s(x) d x} \geq \frac{\int_{\mathbb{R}_{+}^{n}} f(x) s(x) d x}{\int_{\mathbb{R}_{+}^{n}} g(x) s(x) d x},
$$

where again we can assume the left side is well defined.

If we rotate clockwise a line passing through $O_{m}$ in a continuous fashion from the vertical to the horizontal position, and divide $K_{m}$ into two parts $K_{+}$ and $K_{-}$, then the integrals $\int_{K_{+} \times \mathbb{R}_{+}^{n-2}} f(x) s(x) d x$ and $\int_{K_{+} \times \mathbb{R}_{+}^{n-2}} g(x) s(x) d x$ will vary continuously. If for any of the intermediate positions of the line the second of these integrals is zero, we can take $K_{m+1}=K_{-}$as previously. If not, then their quotient varies continuously. For the vertical line we have $K_{+}=K_{E}$, so by (12) the quotient is no larger than for the whole $K_{m}$. For the horizontal line, $K_{+}=K_{S}$, thus by (13) the quotient is no smaller than for the whole $K_{m}$. Thus by the Darboux property there exists a division of $K_{m}$ into two sets $K_{+}$and $K_{-}$, both of which satisfy (6) and (5). Both those sets are spanned.

Notice that at least one of these sets has to satisfy condition (7): if both did not, then $K_{m}$ could not satisfy it either. Let $K_{m+1}$ be such a set.

Obviously $K_{m+1} \subset K_{m}$ and $K_{m+1}$ is a spanned set.

Now let us consider condition (8). Notice that if a point $q$ is used as the point $O_{m}$ for some $m$, then it will lie on the edge of $K_{m+1}$, and thus not in the interior of any $K_{l}$ for $l>m$, and so will not be re-used as $O_{l}$ for $l>m$. Thus no point with both coordinates rational lies in the interior of $K_{\infty}:=\bigcap_{m=0}^{\infty} K_{m}$. Moreover, $K_{\infty}$ is the intersection of a family of convex sets, and thus a convex set, so it has to be an interval or a point (if it contained three affinely independent points, it would contain their convex hull, and inside it a point with both coordinates rational).

As all $K_{m}$ satisfy condition (7), every set $K_{m} \times \mathbb{R}^{n-2}$ has a nonempty intersection with $\mathbb{R}^{n} \backslash A_{t / 2}$ (for otherwise inequality (11) would contradict condition (7)). Thus $K_{\infty} \times \mathbb{R}^{n-2}$ also intersects $\mathbb{R}^{n} \backslash A_{t / 2}$, and thus its intersection with $\mathbb{R}^{n} \backslash A_{t / 3}$ has positive $\left(n-2+\operatorname{dim} K_{\infty}\right)$-dimensional measure, and $K_{\infty} \times \mathbb{R}^{n-2}$ contains a point from the interior of supp $s$. We can thus apply Corollary 2.4 taking $K=K_{\infty}$. We obtain some subsequence $K_{m_{i}}$ of $K_{m}$ and a log-concave function $z$ on $K$ (denoted $g$ in Corollary 2.4).

Let $H$ be the minimal affine subspace containing $K_{\infty} \times \mathbb{R}^{n-2}$. We want to apply the induction assumption to the restrictions of $f, g, h$ and $s \cdot z$ 
to $H$. The subspace $H$ can be given by either $x_{1}=a x_{2}+b$ or $x_{2}=a x_{1}+b$, as $K_{\infty}$ is a spanned set, thus the restriction of $s$ to $H$ is a descendant of $\mathcal{F}$ (with $H$ as domain and an appropriate modification of the basis). Since $z$ is log-concave and given by a single variable on $K$, the function $s \cdot z$ on $H$ is also a descendant of $\mathcal{F}$, thus an Orlicz-based function. The restrictions of $s \cdot z, f$ and $g$ to $H$ satisfy the hereditary $\Theta$ condition, as $s \cdot z$ is a descendant of $s$. Condition (4) is satisfied by going to the limit in conditions (6) and (7) and using Corollary 2.4. Thus all the assumptions are satisfied, and by the induction hypothesis there exists an interval $I$ in $H$ and a log-concave measure $\nu$ as in the conclusion of the lemma; and this interval and measure satisfy the conclusion also for $\mathbb{R}^{n}$, which ends the proof in the general case.

Note that the hereditary $\Theta$ condition implies in particular that $f / g$ is decreasing on $I$. If $h$ were coordinatewise decreasing, we would obtain a contradiction with inequality (4) by the continuous version of the Chebyshev sum inequality.

To end the proof we only need Lemma 2.9, which describes the behaviour of the proportion of integrals of $f$ and $g$ under the hereditary $\Theta$ condition when we divide a spanned set by a horizontal or vertical line. This was basically proved in [6]; the proof is repeated here for the sake of completeness.

Lemma 2.9. Let $\mathcal{F}=\left(s, \mathbb{R}^{n}, \mathcal{E}\right)$, and let $f$ and $g$ be as in the assumptions of Lemma 2.8. Let $K$ be a spanned set in $\operatorname{span}\left\{e_{1}, e_{2}\right\}$ such that $\int_{K \times \mathbb{R}^{n-2}} s(x) d x>0$. Let $K_{x}=K \cap\left\{v: v_{1}=x\right\}$ be the intersection of the set $K$ with a vertical line. Let

$$
\Theta(x)=\frac{\int_{K_{x} \times \mathbb{R}^{n-2}} f(v) s(v) d \lambda_{n-1}(v)}{\int_{K_{x} \times \mathbb{R}^{n-2}} g(v) s(v) d \lambda_{n-1}(v)} .
$$

Then $\Theta(x)$ is decreasing on its domain. In particular, if the line $v_{1}=x_{0}$ divides $K$ into two parts, $K_{-}=\left\{v \in K: v_{1} \leq x_{0}\right\}$ and $K_{+}=\left\{v \in K: v_{1} \geq x_{0}\right\}$, then

$$
\frac{\int_{K_{-} \times \mathbb{R}^{n-2}} f(v) s(v) d v}{\int_{K_{-} \times \mathbb{R}^{n-2}} g(v) s(v) d v} \geq \frac{\int_{K_{+} \times \mathbb{R}^{n-2}} f(v) s(v) d v}{\int_{K_{+} \times \mathbb{R}^{n-2}} g(v) s(v) d v}
$$

if both sides are well defined.

Proof. First note the following simple fact:

FACT 2.10. Let $\mu$ be any measure on the interval $I$. Let $f, g: I \rightarrow \mathbb{R}_{+}$, suppose $\operatorname{supp} f \subset \operatorname{supp} g$ and $f / g$ is decreasing on $\operatorname{supp} g$. Then for any $a<b \leq d$ and $a \leq c<d$ we have

$$
\frac{\int_{a}^{b} f(x) d \mu}{\int_{a}^{b} g(x) d \mu} \geq \frac{\int_{c}^{d} f(x) d \mu}{\int_{c}^{d} g(x) d \mu}
$$

if both sides are well defined. 
From the $\Theta$ property for any $y_{0}$ the function

$$
x \mapsto \frac{\int_{\{x\} \times\left\{y_{0}\right\} \times \mathbb{R}^{n-2}} f(v) s(v) d \lambda_{n-2}(v)}{\int_{\{x\} \times\left\{y_{0}\right\} \times \mathbb{R}^{n-2}} g(v) s(v) d \lambda_{n-2}(v)}
$$

is decreasing where defined. The support of $s$ is convex, $K$ is also convex, $\operatorname{supp} g \supset \operatorname{supp} s$, and thus the domain of this function is an interval. Thus by Fact 2.10 , we obtain

$$
\frac{\int_{\{x\} \times\left[y_{a}, y_{b}\right] \times \mathbb{R}^{n-2}} f(v) s(v) d \lambda_{n-1}(v)}{\int_{\{x\} \times\left[y_{a}, y_{b}\right] \times \mathbb{R}^{n-2}} g(v) s(v) d \lambda_{n-1}(v)} \geq \frac{\int_{\{x\} \times\left[y_{c}, y_{d}\right] \times \mathbb{R}^{n-2}} f(v) s(v) d \lambda_{n-1}(v)}{\int_{\{x\} \times\left[y_{c}, y_{d}\right] \times \mathbb{R}^{n-2}} g(v) s(v) d \lambda_{n-1}(v)}
$$

as long as $y_{a}<y_{b}<y_{d}$ and $y_{a}<y_{c}<y_{d}$ and both sides are well defined.

The second property we need is

$$
\frac{\int_{\left\{x_{1}\right\} \times\left[y_{a}, y_{b}\right] \times \mathbb{R}^{n-2}} f(v) s(v) d \lambda_{n-1}(v)}{\int_{\left\{x_{1}\right\} \times\left[y_{a}, y_{b}\right] \times \mathbb{R}^{n-2}} g(v) s(v) d \lambda_{n-1}(v)} \geq \frac{\int_{\left\{x_{2}\right\} \times\left[y_{a}, y_{b}\right] \times \mathbb{R}^{n-2}} f(v) s(v) d \lambda_{n-1}(v)}{\int_{\left\{x_{2}\right\} \times\left[y_{a}, y_{b}\right] \times \mathbb{R}^{n-2}} g(v) s(v) d \lambda_{n-1}(v)}
$$

as long as $x_{2}>x_{1}$ and both sides are well defined. To see this, notice that by moving the origin to $\left(0, y_{a}, 0, \ldots, 0\right)$ and multiplying $s$ by $\mathbf{1}_{y \leq y_{b}}$ we obtain a descendant of $\mathcal{F}$, and thus the hereditary $\Theta$ condition guarantees in particular

$$
\begin{aligned}
\begin{array}{l}
\int_{\left\{x_{1}\right\} \times \mathbb{R} \times \mathbb{R}^{n-2}} f(v) m(v) \widetilde{s}(v) d \lambda_{n-1}(v) \\
\int_{\left\{x_{1}\right\} \times \mathbb{R} \times \mathbb{R}^{n-2}} g(v) m(v) \widetilde{s}(v) d \lambda_{n-1}(v)
\end{array} \\
\quad \geq \frac{\int_{\left\{x_{2}\right\} \times \mathbb{R} \times \mathbb{R}^{n-2}} f(v) m(v) \widetilde{s}(v) d \lambda_{n-1}(v)}{\int_{\left\{x_{2}\right\} \times \mathbb{R} \times \mathbb{R}^{n-2}} g(v) m(v) \widetilde{s}(v) d \lambda_{n-1}(v)},
\end{aligned}
$$

which gives the above property.

Now notice that as $K$ is spanned, for $x_{2}>x_{1}$ we have $K_{x_{1}}=\left\{x_{1}\right\} \times$ $\left[y_{a}, y_{b}\right], K_{x_{2}}=\left\{x_{2}\right\} \times\left[y_{c}, y_{d}\right]$ and $y_{a}<y_{b}<y_{d}$ and $y_{a}<y_{c}<y_{d}$, which gives the first part of the conclusion. The second follows from the first and Fact 2.10.

3. Negative association of absolute values for Orlicz balls. We shall use Lemma 2.8 to prove negative association of absolute values for Orlicz balls. This section closely follows the arguments of [6]. We shall need a pair of functions satisfying the $\Theta$ condition.

Lemma 3.1. Let $\mathcal{F}=(s, V, \mathcal{E})$ be an Orlicz-based function, and let $V=$ $W \times \mathbb{R}, \mathcal{E}=\mathcal{E}^{\prime} \cup\left\{e_{n}\right\}$ be a splitting of $V$ with respect to $\mathcal{E}$. Let $f(x)=s\left(x, z_{2}\right)$ and $g(x)=s\left(x, z_{1}\right)$ for some numbers $0<z_{1}<z_{2}$ and $x \in W$, and suppose $\operatorname{supp} g$ is nonempty. Let $\mathcal{G}=\left(t, W, \mathcal{E}^{\prime}\right)$ be an oriented function, where

$$
t(x):=\mathbf{1}_{\text {supp } g}(x) \prod_{i=1}^{\operatorname{dim} W} u_{i}\left(x_{i}\right)
$$


for some log-concave functions $u_{i}$. Then $\mathcal{G}$ is an Orlicz-based function and $\mathcal{G}$, $f$ and $g$ satisfy the hereditary $\Theta$ condition.

Proof. Let $n=\operatorname{dim} V$. First we shall check that $\mathcal{G}$ is an Orlicz-based function. Let $\left(w_{i}\right)_{i=1}^{n},\left(f_{i}\right)_{i=1}^{n}$ and $m$ be functions witnessing that $\mathcal{F}$ is an Orlicz-based function. Consider the following functions on $W:\left(u_{i} \phi\left(w_{i}\right)\right)_{i=1}^{n-1}$, $\left(f_{i}\right)_{i=1}^{n-1}$ and $x \mapsto \phi\left(m\left(x+f_{n}\left(z_{1}\right)\right)\right)$, where $\phi=\mathbf{1}_{(0, \infty)}$. These functions show $\mathcal{G}$ is an Orlicz-based function.

We proceed to prove the $\Theta$ condition. We will consider the splitting $W=W_{1} \oplus W_{2}$; let $\operatorname{dim} W_{1}=k$. We want to check that the function

$$
\frac{\int_{W_{2}} f(x, y) t(x, y) d \lambda_{n-k-1}(y)}{\int_{W_{2}} g(x, y) t(x, y) d \lambda_{n-k-1}(y)}
$$

is coordinatewise nonincreasing on $W_{1}$. Obviously it suffices to change one coordinate at a time, keeping the others fixed. Notice that fixing the coordinate $x_{i}$ is equivalent to intersecting $W$ with a subspace given by $x_{i}=b$ and replacing $\mathcal{F}$ and $\mathcal{G}$ by their appropriate descendants (and, correspondingly, $f$ and $g$ by their appropriate restrictions), and thus by simple induction upon $\operatorname{dim} W_{1}$ it suffices to consider the case $\operatorname{dim} W_{1}=1$. Without loss of generality we can identify $W_{2}$ with $\mathbb{R}^{n-2}$. Thus it suffices to prove, for $0<x_{1}<x_{2}$,

$$
\begin{aligned}
& \int_{\mathbb{R}^{n-2}} s\left(x_{1}, y, z_{1}\right) t\left(x_{1}, y\right) d \lambda_{n-2}(y) \int_{\mathbb{R}^{n-2}} s\left(x_{2}, y, z_{2}\right) t\left(x_{2}, y\right) d \lambda_{n-2}(y) \\
& \leq \int_{\mathbb{R}^{n-2}} s\left(x_{1}, y, z_{2}\right) t\left(x_{1}, y\right) d \lambda_{n-2}(y) \int_{\mathbb{R}^{n-2}} s\left(x_{2}, y, z_{1}\right) t\left(x_{2}, y\right) d \lambda_{n-2}(y) .
\end{aligned}
$$

Notice that as

$$
\begin{aligned}
s\left(x_{i}, y, z_{j}\right)= & m\left(f_{1}\left(x_{i}\right)+f_{2}\left(y_{1}\right)+\cdots+f_{n-1}\left(y_{n-2}\right)+f_{n}\left(z_{j}\right)\right) \\
& \times w_{1}\left(x_{i}\right) w_{n}\left(z_{j}\right) \prod_{i=2}^{n-1} w_{i}\left(y_{i-1}\right)
\end{aligned}
$$

the expressions $w_{1}\left(x_{i}\right)$ and $w_{n}\left(z_{j}\right)$ cancel out in (14). Similarly the $u_{1}\left(x_{i}\right)$ expressions in $t$ cancel out, and we can also drop the $\mathbf{1}_{\text {supp } g}$ factor from $t$ as all the integrands disappear outside $\operatorname{supp} g$. Now consider

$$
\begin{aligned}
r\left(y_{0}, y_{1}, \ldots, y_{n-2}\right)= & m\left(y_{0}+f_{2}\left(y_{1}\right)+f_{3}\left(y_{2}\right)+\cdots+f_{n-1}\left(y_{n-2}\right)\right) \\
& \times \prod_{i=1}^{n-2} w_{i+1}\left(y_{i}\right) u_{i+1}\left(y_{i}\right) .
\end{aligned}
$$

This function is log-concave, since the composition of an increasing convex function with a convex function is convex and the product of log-concave functions is log-concave. Let $P_{x}=\int_{\mathbb{R}^{n-2}} r(x, v) d \lambda_{n-2}(v)$. As $r$ is log-concave, by the Prekopa-Leindler inequality (see [3]) we have $P_{x}^{t} P_{y}^{1-t} \leq P_{t x+(1-t) y}$. 
Let $a=f_{1}\left(x_{1}\right)+f_{n}\left(z_{1}\right), b=f_{1}\left(x_{2}\right)-f_{1}\left(x_{1}\right)$ and $c=f_{n}\left(z_{2}\right)-f_{n}\left(z_{1}\right)$. In particular,

$$
\begin{aligned}
& P_{a}^{c /(b+c)} P_{a+b+c}^{b /(b+c)} \leq P_{a+b}, \\
& P_{a}^{b /(b+c)} P_{a+b+c}^{c /(b+c)} \leq P_{a+c},
\end{aligned}
$$

and thus

$$
P_{a} P_{a+b+c} \leq P_{a+b} P_{a+c},
$$

which proves inequality (14), and thus the conclusion.

Finally, we prove the hereditary $\Theta$ condition. Let $\mathcal{G}^{\prime}=\left(t^{\prime}, W^{\prime}, \mathcal{E}^{\prime}\right)$ be a descendant of $\mathcal{G}$. We will construct an Orlicz-based function $\mathcal{F}^{\prime}=\left(s^{\prime}, W^{\prime} \times \mathbb{R}\right.$, $\left.\mathcal{E}^{\prime} \cup\left\{e_{0}\right\}\right)$ and log-concave functions $u_{i}^{\prime}$ so that $f$ restricted to $W^{\prime}$ is equal to $s\left(\cdot, z_{1}\right), g$ restricted to $W^{\prime}$ is $s\left(\cdot, z_{2}\right)$, and $t^{\prime}=\operatorname{supp} g \prod u_{i}^{\prime}$.

The construction is by induction upon the descendant hierarchy. Thus suppose $\mathcal{G}^{\prime}$ is a son of $\mathcal{G}$. We have to consider three cases:

- If $t^{\prime}(x)=t(x) w\left(x_{i}\right)$, take $u_{i}^{\prime}=u_{i} \cdot w$ and leave all other parameters unchanged.

- If $W^{\prime}$ is given by $a x_{j}+b$, take as $\mathcal{F}^{\prime}$ the son of $\mathcal{F}$ given by the same equation $x_{i}=a x_{j}+b$, and replace $u_{i}, u_{j}$ by a single function $u(t)=$ $u_{i}(a t+b) u_{j}(t)$.

- If the origin is moved to $x$, consider the son of $\mathcal{F}$ given by moving the origin to $(x, 0)$, and the functions $u_{i}^{\prime}(t)=u_{i}\left(t+x_{i}\right)$.

In each case we can proceed with the inductive construction, and having constructed $\mathcal{F}^{\prime}$ and $u_{i}^{\prime}$ we apply the previous result.

Lemma 3.2. Let $\mathcal{F}=(s, V, \mathcal{E})$ be an Orlicz-based function, and let $V=$ $W \times \mathbb{R}, \mathcal{E}=\mathcal{E}^{\prime} \cup\left\{e_{n}\right\}$ be a splitting of $V$ with respect to $\mathcal{E}$. Let $h: W_{+} \rightarrow \mathbb{R}$ be a bounded coordinatewise decreasing function, and let $0<z_{1}<z_{2}$. Let $\mathcal{G}=(t, W, \mathcal{E})$ be an oriented function, where

$$
t(x)=\mathbf{1}_{\left\{s\left(x, z_{1}\right)>0\right\}} \prod u_{i}\left(x_{i}\right),
$$

where the $u_{i}$ are log-concave functions. Then

$$
\frac{\int_{W_{+}} h(x) s\left(x, z_{2}\right) t(x) d \lambda_{n-1}(x)}{\int_{W_{+}} h(x) s\left(x, z_{1}\right) t(x) d \lambda_{n-1}(x)} \geq \frac{\int_{W_{+}} s\left(x, z_{2}\right) t(x) d \lambda_{n-1}(x)}{\int_{W_{+}} s\left(x, z_{1}\right) t(x) d \lambda_{n-1}(x)}
$$

if both sides are well defined.

Proof. Suppose the conclusion does not hold. Then for some fixed $h$,

$$
\frac{\int_{W_{+}} h(x) s\left(x, z_{2}\right) t(x) d \lambda_{n-1}(x)}{\int_{W_{+}} h(x) s\left(x, z_{1}\right) t(x) d \lambda_{n-1}(x)}<\frac{\int_{W_{+}} s\left(x, z_{2}\right) t(x) d \lambda_{n-1}(x)}{\int_{W_{+}} s\left(x, z_{1}\right) t(x) d \lambda_{n-1}(x)} .
$$

We would like to apply Lemma 2.8 with the role of $f$ played by $s\left(x, z_{2}\right)$, and the role of $g$ by $s\left(x, z_{1}\right)$. For this we need $s\left(x, z_{1}\right), s\left(x, z_{2}\right)$ and $h$ to be 
continuous and $h$ to be bounded uniformly away from zero. First, notice that if inequality (15) holds, then it will also hold if we substitute $h(x)+C$ for $h$. Thus we may assume $h$ is strictly larger than, say, 1 . Since we have a strict inequality in (15), it will also hold after a small enough modification of $s$ and $h$. Let $s(v)=m\left(\sum f_{i}\left(v_{i}\right)\right) \prod w_{i}\left(v_{i}\right)$, where $v=(x, z)$. First we approximate $m$ from above by a decreasing sequence $m_{k}$ of continuous log-concave functions with maxima at zero, which converges pointwise to $m$. Then $s_{k}\left(x, z_{i}\right)$ converges monotonically to $s\left(x, z_{i}\right)$, and thus all the integrals in (15) converge and we can choose a $k$ such that (15) still holds after substituting $s_{k}$ for $s$. Similarly we can approximate $f_{i}$ from below by continuous Young functions (i.e. functions that do not jump to $\infty$ ). Then $s_{k}$ will still be an Orlicz-based function, and $f$ and $g$ will be continuous and satisfy supp $f \subset \operatorname{supp} g$ (as $f \leq g$, because both the Young functions and $m$ are increasing). Similarly we approximate $h$ from above by a sequence of continuous functions $h_{k}$, decreasing coordinatewise and uniformly bounded away from zero and pointwise convergent to $h$, and replace $h$ by a sufficiently close approximation $h_{k}$. We can assume that (15) still holds after these modifications.

After the modifications the assumptions of Lemma 2.8 are satisfied: the hereditary $\Theta$ condition holds by Lemma 3.1, as $s_{k}$ is an Orlicz-based function, and condition (4) is simply the inequality (15). Thus the conclusion of Lemma 2.8 holds - but we assumed $h_{k}$ to be coordinatewise decreasing, and this contradiction ends the proof.

Notice that from the above lemma, by switching sides we deduce that the function

$$
\frac{\int_{\mathbb{R}_{+}^{k}} h(x) s(x, z) t(x) d x}{\int_{\mathbb{R}_{+}^{k}} s(x, z) t(x) d x}
$$

is coordinatewise decreasing as a function of $z$ for any coordinatewise decreasing function $h$ and any Orlicz-based function $\mathcal{F}$ on $\mathbb{R}^{n}$. Thus we can prove the following corollary just as we proved the last part of Lemma 3.1:

Corollary 3.3. Consider $\mathcal{F}, \mathcal{G}$, and $h$ defined as above. Then the Orlicz-based function $\mathcal{G}$ and the functions $\int_{W_{+}} h(x) s\left(x, z_{1}\right) t(x) d \lambda_{n-1}(x)$ and $\int_{W_{+}} s\left(x, z_{1}\right) t(x) d \lambda_{n-1}(x)$ satisfy the hereditary $\Theta$ condition.

Now we can prove our statement in full generality:

LEMma 3.4. Let $\varphi=\left(s, \mathbb{R}^{n}, \mathcal{E}\right)$ be an Orlicz-based function, and let $h$ : $\mathbb{R}_{+}^{k} \rightarrow \mathbb{R}$ and $\bar{h}: \mathbb{R}_{+}^{n-k} \rightarrow \mathbb{R}$ be coordinatewise decreasing functions. Then

$$
\frac{\int_{\mathbb{R}_{+}^{n-k}} \bar{h}(x) \int_{\mathbb{R}_{+}^{k}} h(y) s(x, y) d y d x}{\int_{\mathbb{R}_{+}^{n-k}} \bar{h}(x) \int_{\mathbb{R}_{+}^{k}} s(x, y) d y d x} \leq \frac{\int_{\mathbb{R}_{+}^{n-k}} \int_{\mathbb{R}_{+}^{k}} h(y) s(x, y) d y d x}{\int_{\mathbb{R}_{+}^{n-k}} \int_{\mathbb{R}_{+}^{k}} s(x, y) d y d x} .
$$


The proof will be almost identical to the proof of Lemma 3.2:

Proof. Again we apply Lemma 2.8. Assume the opposite inequality holds. The role of the function $h$ will be taken, as in Lemma 3.2, by a continuous and coordinatewise decreasing approximation of $\bar{h}$, uniformly bounded from below. We define $f(x)=\int_{\mathbb{R}_{+}^{k}} h(y) s(x, y) d y$ and $g(x)=\int_{\mathbb{R}_{+}^{k}} s(x, y) d y$. Approximating $m, f_{i}$ and $\bar{h}$ by continuous functions as in 3.2 we obtain continuous modifications of $f$ and $g$, for which (4) still holds. The hereditary $\Theta$ condition is satisfied by Corollary 3.3. However, our function $h$ is coordinatewise decreasing, which contradicts Lemma 2.8.

From the above we immediately obtain Theorem 1.3.

Acknowledgements. This research was partially supported by MEiN Grant no 1 PO3A 01229 and by the Polish Foundation for Science.

\section{References}

[1] K. Alam and K. M. L. Saxena, Positive dependence in multivariate distributions, Comm. Statist. Theory Methods A 10 (1981), 1183-1196.

[2] B. Fleury, Between Paouris concentration inequality and variance conjecture, preprint.

[3] R. J. Gardner, The Brunn-Minkowski inequality, Bull. Amer. Math. Soc. 39 (2002), 355-405.

[4] K. Joag-Dev and F. Proschan, Negative association of random variables with applications, Ann. Statist. 11 (1983), 286-295.

[5] R. Kannan, L. Lovász and M. Simonovits, Isoperimetric problems for convex bodies and a localization lemma, Discrete Comput. Geom. 13 (1995), 541-559.

[6] M. Pilipczuk and J. O. Wojtaszczyk, The negative association property for the absolute values of random variables equidistributed on a generalized Orlicz ball, Positivity 12 (2008), 421-474.

[7] J. O. Wojtaszczyk, The square negative correlation property for generalized Orlicz balls, in: Geometric Aspects of Functional Analysis (Israel Seminar, 2004-2005), Lecture Notes in Math. 1910, Springer, 2007, 305-313.

Jakub Onufry Wojtaszczyk

Department of Mathematics,

Computer Science and Mechanics

University of Warsaw

Banacha 2

02-097 Warszawa, Poland

E-mail: onufry@duch.mimuw.edu.pl

Received November 25, 2008;

received in final form April 4, 2009 